\title{
Neurofibromatosis-associated lung disease: a case series and literature review
}

\author{
A.C. Zamora*,\#,+, H.R. Collard*,\#, P.J. Wolters ${ }^{\#}$, W.R. Webb" and T.E. King*,\#
}

ABSTRACT: An association of neurofibromatosis with diffuse lung disease (NF-DLD) has been described, but its true prevalence and characteristics remain unclear. The objective of the present study was to define diffuse lung disease in patients with neurofibromatosis.

A retrospective case series and literature review in a tertiary care academic medical centre is reported in which medical records, chest radiographs and high-resolution computed tomography (HRCT) scans were reviewed.

A total of 55 adult patients with neurofibromatosis were identified, three of whom had NF-DLD. A literature review revealed 16 articles reporting 61 additional cases, yielding a total of 64 NF-DLD cases. The mean age of patients was 50 yrs. Males outnumbered females; most reported dyspnoea. Of the 16 subjects with documented smoking histories, 12 were ever-smokers. Eight patients had HRCT scan results demonstrating ground-glass opacities (37\%), bibasilar reticular opacities $(50 \%)$, bullae $(50 \%)$, cysts $(25 \%)$ and emphysema $(25 \%)$; none had honeycombing. A group of 14 patients had surgical biopsy results that showed findings of interstitial fibrosis (100\%) and interstitial inflammation (93\%).

In conclusion, neurofibromatosis with diffuse lung disease is a definable clinical entity, characterised by upper lobe cystic and bullous disease and lower lobe fibrosis. Its relationship to smoking remains unclear.

KEYWORDS: Cystic lung disease, high-resolution computed tomography, interstitial lung disease, neurofibromatosis, pulmonary fibrosis

V on Recklinhausen's disease or neurofibromatosis type 1 (NF) is an autosomal dominant dysplasia of ectoderm and mesoderm with a variable clinical expression characterised by collections of neurofibromas, café-au-lait spots and pigmented hamartomas in the iris (Lish nodules) [1]. NF has a prevalence of one in 3,000 and in $30-50 \%$ of cases there is no family history of the disease. These sporadic cases probably arise from (usually paternal) germ cell mutations [1].

In NF, the thorax and lungs can be affected in several ways: cutaneous and subcutaneous neurofibromas on the chest wall; kyphoscoliosis; ribbon deformity of the ribs; thoracic neoplasms; and interstitial lung disease (ILD) [1]. Sporadic cases of NF with diffuse lung disease (NF-DLD) have been published in case reports, but the overall prevalence and clinical characteristics of NF-DLD remain unclear. A recent article questioned the association of NF with diffuse ILD [2].
The present study reports three patients with NF-DLD seen at the University of California, San Francisco, CA, USA. The clinical and radiographic characteristics of cases of NF-DLD reported in the literature are also summarised.

\section{METHODS}

Medical records of all adult patients (aged $>25$ yrs) with NF seen at the University of California between 1980 and 2004 were reviewed for evidence of DLD. NF was defined according to published diagnostic criteria [3]. Only adult cases of NF-DLD were included in the present study as it has been reported previously that NFDLD does not occur in children. Primary radiographic data were obtained on identified cases of NF-DLD and reviewed by an experienced pulmonary radiologist (W.R. Webb). A literature review was performed using PubMed (keywords "neurofibroma", "fibrosis", "scar", "pulmonary", "interstitial lung disease", and "lung"), by searching references of published articles and through

\section{AFFILIATIONS}

*Dept of Medicine, San Francisco General Hospital, and Depts of ${ }^{\#}$ Medicine, and

-Radiology, University of California San Francisco, San Francisco, CA USA.

+University Hospital "Dr. Jose Eleuterio González", Monterrey, NL, Mexico.

CORRESPONDENCE

T.E. King

San Francisco General Hospital

1001 Potrero Avenue

Room $5 \mathrm{H} 22$

San Francisco

CA 94110

USA

Fax: 14152064890

E-mail: tking@medsfgh.ucsf.edu

Received:

March 292006

Accepted after revision:

July 132006 
input from experts in the field. Articles reporting cases of NFDLD were included in the present review.

Data from newly identified cases were combined with data abstracted from identified articles. Variables extracted were: age; sex; dyspnoea; cough; chest pain; smoking status; pulmonary function pattern (obstructive or restrictive); diffusing capacity of the lung for carbon monoxide (DL,CO; normal or decreased); high-resolution computed tomography (HRCT) patterns (ground glass, linear densities, nodular densities, bullae and honeycombing); surgical lung biopsy (yes or no); and histopathological patterns (fibrosis, inflammation and honeycombing). For some variables of interest, only aggregate data were available. Statistics were calculated for each variable: age was reported as mean (because of aggregate data, the SD could not be determined). All other variables were reported as percentages. Cases of known nonsmokers were compared to cases of known smokers using univariate linear regression for continuous variables and Fisher's exact test for dichotomous variables.

\section{RESULTS}

Among 55 cases of NF seen at the University of California between 1980 and 2004 (prevalence 5.5\%), three patients were identified with NF-DLD. These cases are described below, summarised in table 1 and illustrated in figures 1-3.

\section{Case 1}

A 66-yr-old Caucasian male with NF was referred for evaluation of severe dyspnoea. He was unable to walk $>15.24 \mathrm{~m}$ without stopping. He was a former smoker. A chest examination revealed diffuse inspiratory crackles. There was no clubbing. Pulmonary function testing is shown in table 1. HRCT of the chest showed diffuse and numerous small rounded thin-walled lung cysts with an upper lobe predominance (fig. 1).

\section{Case 2}

A 53-yr-old Caucasian male with NF was referred for evaluation for lung transplantation. He had chronic cough and dyspnoea. He was a former smoker. Chest examination was normal. Pulmonary function testing is shown in table 1. HRCT revealed bilateral asymmetric bullous disease with an apical predominance (fig. 2).

\section{Case 3}

A 70-yr-old African-American female with NF was referred for evaluation of chest pain. She had never smoked. Chest examination was normal. No pulmonary function testing was performed. HRCT showed scattered small round lung cysts in both lungs with an apical predominance (fig. 3).

\section{Literature review}

The literature review revealed 529 articles, which were reviewed by one of the current authors (A.C. Zamora). In total, 16 articles were identified reporting 61 cases of NF-DLD. Combined with three newly identified cases, a total of 64 cases of NF-DLD have now been reported (table 2). The mean (range) age of patients was 50 (23-72) yrs. There were 44 males $(69 \%)$ and $20(31 \%)$ females. Common symptoms include dyspnoea $(80 \%)$ and cough $(32 \%)$. Only $5 \%$ presented with chest pain and $11 \%$ were asymptomatic.

Pulmonary function results were available in 30 cases and in all but one case (3\%) the function was abnormal. An obstructive pattern was seen in $43 \%$ of patients, $37 \%$ had a restrictive pattern and $17 \%$ had a mixed pattern. When measured $(n=18)$, DL,CO was almost always decreased $(94 \%$ of cases).

Chest radiograph readings were available in 63 cases and HRCT was available in eight cases (table 3). On chest radiography, bullous lung disease was present in $73 \%$ of cases, almost always in the upper lobes (93\%). Basilar linear densities were present in $62 \%$ and radiographic honeycombing in $13 \%$ of cases. HRCT revealed emphysema (25\%), cyst (25\%), ground-glass abnormality (37\%), bullae (50\%) and reticular abnormalities (50\%).

A total of 16 cases had smoking histories available. Of these, four $(25 \%)$ were nonsmokers. When data were analysed by smoking status, nonsmokers were more likely to be female $(100 \%$ versus $25 \%, p=0.01)$. There were no statistically significant differences in age, pulmonary function pattern or radiographic abnormalities between nonsmokers and smokers (table 4).

\section{DISCUSSION}

NF-associated ILD was first described in 1963 by DAVIES [4] and, over the ensuing decades, other reports have described the association between NF and ILD [4-18]. In some patients,

\section{TABLE 1 New cases of neurofibromatosis with diffuse lung disease}

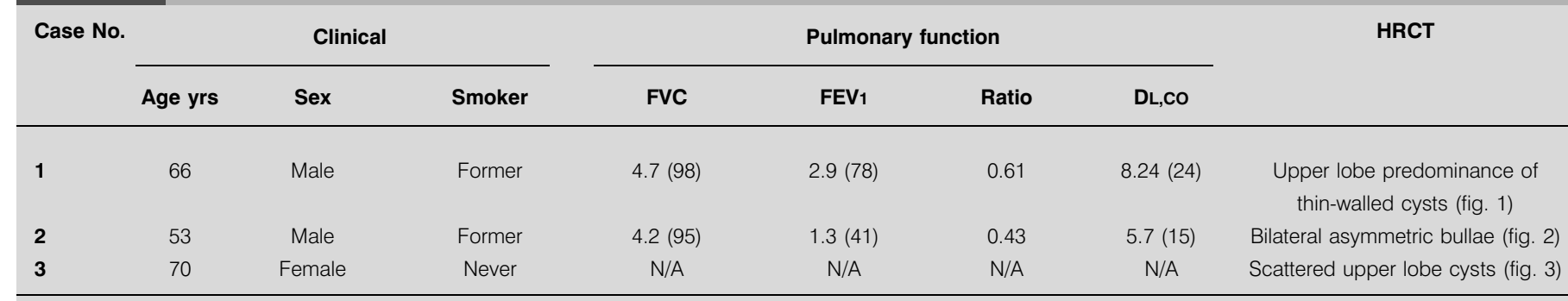

Data are presented as $\mathrm{n}$ or $\mathrm{n}(\%)$. HRCT: high-resolution computed tomography; FVC: forced vital capacity; FEV1: forced expiratory volume in one second; DL,CO: diffusing capacity of the lung for carbon monoxide; N/A: not applicable. 


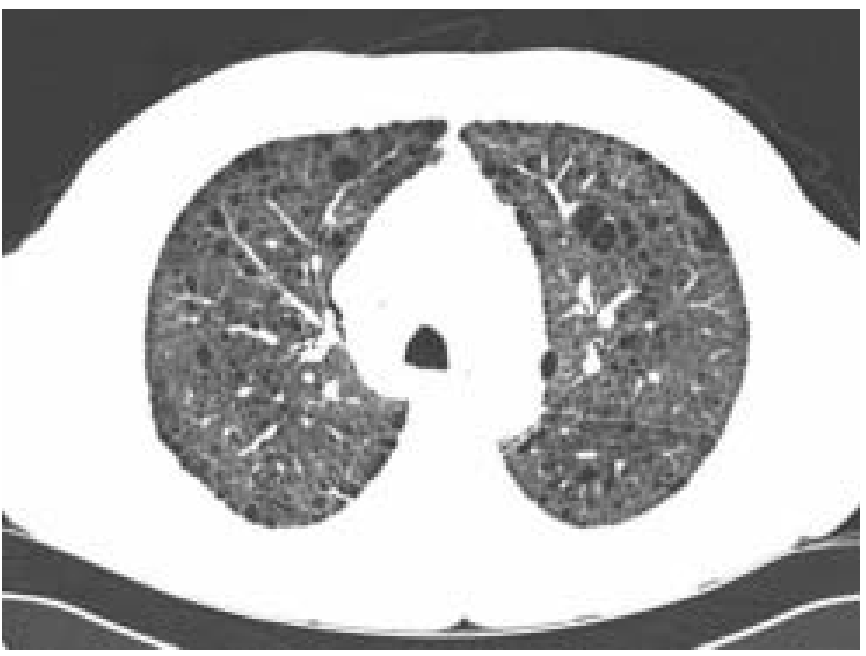

FIGURE 1. High-resolution computed tomography from case No. 1 shows diffuse, numerous, small rounded lung cysts involving the upper lobes and superior segment of the left lower lobe. In distinction to centrilobular emphysema, the cysts have well-defined walls.

the finding of lung disease was incidental, but most subjects reported dyspnoea on exertion. Pulmonary function tests show either an obstructive or a restrictive defect, and a decreased DL,CO is almost always present.

Radiographic studies of NF patients using conventional chest radiography commonly report large apical asymmetric thinwalled bullae $(73 \%)$, sometimes occupying a substantial portion of hemithorax and associated with areas of hypovascularity and bibasilar, subpleural reticular abnormality (61\%). Honeycombing mimicking idiopathic pulmonary fibrosis is rare but has been described [17]. Limited data are available regarding HRCT appearance in NF-DLD. There are five cases with HRCT imaging reported in the literature and the present
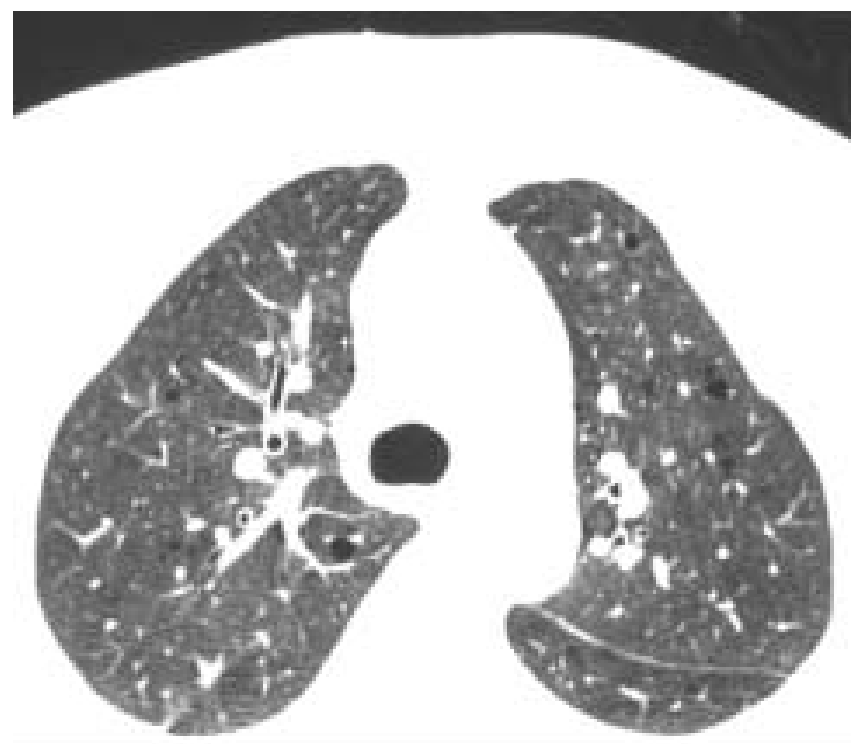

FIGURE 3. High-resolution computed tomography from case No. 3 shows scattered small, round lung cysts in both upper lobes.
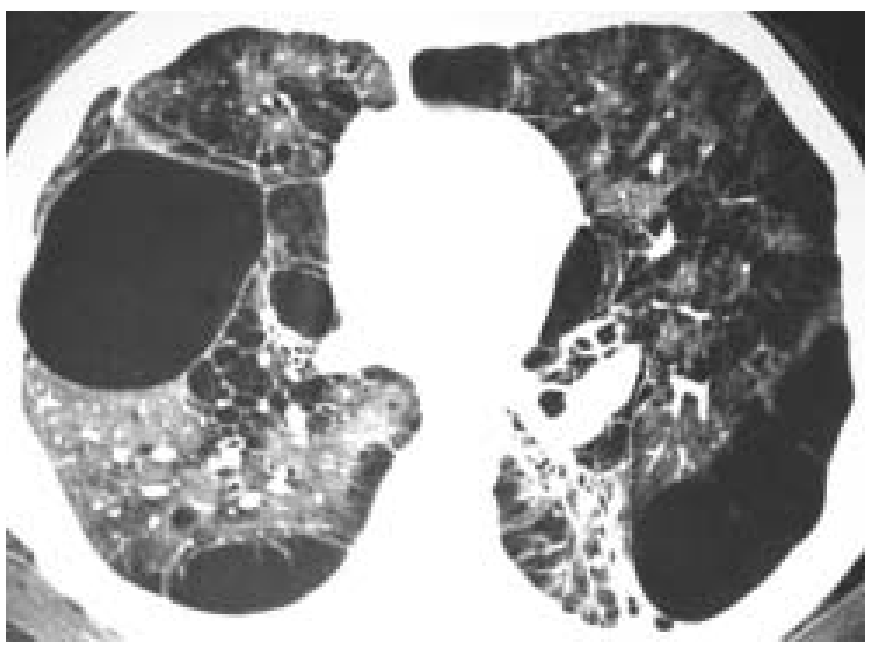

FIGURE 2. High-resolution computed tomography from case No. 2 shows bilateral asymmetric bullous disease.

study reports three additional cases. The current cases revealed cystic changes and bullae, changes distinct from, but easily confused with, emphysema.

There remains debate as to whether NF-DLD is a primary manifestation of NF. RICCARDI [1] did not observe pulmonary parenchymal disease in over 200 patients with various forms of NF, although a HRCT scan was not performed in these patients. Therefore, cases of NF-DLD may have been missed. RYU et al. [2] reviewed 70 patients with NF and found 12 with evidence of NF-DLD; 10 (six by chest radiography and four by $\mathrm{CT}$ ) had emphysema, cystic airspace disease or bullae, and all were current or former smokers. As all of these cases had a history of smoking, it was suggested that these 10 cases may simply represent smoking-related emphysema. Of the 70 cases, three cases had reticular opacities and other potential causes of

\section{TABLE 2 Demographic, clinical and laboratory data}

Variable

$\begin{array}{lc}\text { Subjects n } & 64 \\ \text { Demographics } & \\ \text { Age yrs* } & 50(23-72) \\ \text { Male/female } & 44 / 20 \\ \text { Symptoms }{ }^{\#} & \\ \text { Dyspnoea } & 28(80) \\ \text { Cough } & 11(32) \\ \text { Chest pain } & 2(5) \\ \text { Asymptomatic } & 4(11) \\ \text { Pulmonary function pattern } & \\ \text { Normal } & 1(3) \\ \text { Obstructive } & 13(43) \\ \text { Restrictive } & 11(37) \\ \text { Mixed } & 5(17) \\ \text { Low DL,co } & \end{array}$

Data are presented as $n$, mean (range) or $n(\%)$. DL,CO: diffusing capacity of the lung for carbon monoxide. ${ }^{\#}: \mathrm{n}=35 ; " \mathrm{n}=30 ;{ }^{+}: \mathrm{n}=18$. 


\section{TABLE 3 Radiographic data}

\begin{tabular}{ll} 
Modality & \\
\hline Chest radiograph $^{\#}$ & \\
Mottled & $20(32)$ \\
Linear densities & $38(61)$ \\
Nodular densities & $13(13)$ \\
Bullae & $46(73)$ \\
Bullae upper localisation & $41(93)$ \\
Honeycombing & $7(12)$ \\
HRCT & \\
Ground glass & $3(37)$ \\
Reticular abnormality & $4(50)$ \\
Bullae & $4(50)$ \\
Emphysema & $2(25)$ \\
Cyst & $2(25)$ \\
Honeycombing & $0(0)$ \\
\hline
\end{tabular}

Data are presented as $\mathrm{n}(\%)$. HRCT: high-resolution computed tomography. ${ }^{*}$ $\mathrm{n}=63 ; \bullet: \mathrm{n}=8$.

their diffuse lung disease: residual scarring from acute respiratory distress syndrome; rheumatoid arthritis-related ILD and smoking-related ILD (e.g. desquamative interstitial pneumonia).

Several observations, however, suggest that NF-DLD is a distinct clinical entity. First, of the 16 cases of NF-DLD with recorded smoking histories, four $(25 \%)$ were nonsmokers [5, $12,15]$. Secondly, the radiographic appearance of HRCT in the current three cases (which constitute approximately $40 \%$ of

\section{TABLE 4 Comparison of smokers and nonsmokers}

\begin{tabular}{lccc} 
Variable & Smokers & Nonsmokers & p-value \\
\hline $\begin{array}{l}\text { Subjects n } \\
\text { Demographic }\end{array}$ & 12 & 4 & \\
$\quad$ Age yrs & & & \\
$\quad$ Male/female & $52.4 \pm 9.2$ & $46.75 \pm 20.5$ & 0.4 \\
Pulmonary function testing & $9 / 3(75 / 25)$ & $0 / 4(0 / 100)$ & 0.01 \\
$\quad$ Obstructive pattern & 7 & 2 & \\
Chest radiograph & $7(100)$ & $2(100)$ & 0.5 \\
Linear densities & 12 & 3 & \\
$\quad$ Bullae & $10(90)$ & $2(50)$ & 0.4 \\
HRCT & $11(91)$ & $0(0)$ & 1.0 \\
Ground-glass opacities & 6 & 2 & \\
Reticular & $3(50)$ & $0(0)$ & 0.5 \\
Bullae & $3(50)$ & $1(50)$ & 1.0 \\
Cyst & $3(50)$ & $1(50)$ & 1.0 \\
Emphysema & $1(20)$ & $1(50)$ & 1.0 \\
\hline
\end{tabular}

Data are presented as $n$, mean \pm SD or $n(\%)$. HRCT: high-resolution computed tomography. Surgical lung biopsy results were available in 14 cases, all 14 showed fibrosis and 13 showed interstitial inflammation (93\%). Honeycomb cysts were present in three cases (23\%). reported HRCT cases) is atypical for smoking-related disease; the borders of the cysts and bullae are thicker and more sharply defined than is seen in smoking-related emphysema. Ground-glass opacities, a hallmark of smoking-related ILD, was only seen in three patients $[19,20]$. Thirdly, surgical lung biopsy reports do not describe the intra-alveolar accumulation of pigmented macrophages that would be expected in desquamative interstitial pneumonia or respiratory bronchiolitis associated-ILD [10]. Instead, the alveolar septa demonstrate lymphoplasmocytic inflammation and fibrosis consistent with nonspecific idiopathic pneumonia (NSIP) pattern.

There is biological rationale for a relationship between NF and ILD. PATCHEFSKY et al. [14] suggested that the pulmonary parenchymal disease in NF is attributed to a mesenchymal defect, resulting in primary deposition of collagen. Consistent with this suggestion, the pathological features of the pulmonary parenchymal lesions at autopsy or lung biopsy, reveal areas of normal lung alternating with patchy interstitial fibrosis, septal thickening and cellularity. Moreover, there is perhaps a fibrotic environment in these patients; FABRICANT and TODARO [21] found increased nerve growth factor in the serum of patients with NF. This factor directly activates fibroblasts, stimulating differentiation into more pro-fibrogenic myofibroblasts, a process which may contribute to the pathogenesis of lung fibrosis in NF [22].

The occurrence of NF-DLD in nonsmokers, the presence of cysts distinct from smoking-related emphysema on HRCT and the histopathological pattern of NSIP, all support the association of cystic lung disease as a distinct manifestation of NF. However, it has been suggested that NF may increase the sensitivity of the lungs to cigarette smoke, causing the early development of emphysema-like changes. This suggests smoking may indeed be a risk factor for the development and severity of NF-DLD $[2,18]$.

In summary, the present authors believe currently available data suggest that neurofibromatosis with diffuse lung disease is a distinct clinical entity, characterised by upper lobe cystic and bullous disease and basilar fibrosis. To properly and definitively address the association of neurofibromatosis, diffuse lung disease and cigarette smoking, a cross-sectional cohort study is needed in which patients with neurofibromatosis undergo high-resolution computed tomography and have careful medical histories taken. It is hoped that a better understanding of neurofibromatosis with diffuse lung disease, together with an increased understanding of the pathobiology of neurofibromatosis, will lead to future therapies for this rare but highly morbid condition.

\section{REFERENCES}

1 Riccardi VM. Von Recklinghausen neurofibromatosis. $N$ Engl J Med 1981; 305: 1617-1627.

2 Ryu JH, Parambil JG, McGrann PS, Aughenbaugh GL. Lack of evidence for an association between neurofibromatosis and pulmonary fibrosis. Chest 2005; 128: 2381-2386.

3 Neurofibromatosis. Conference statement. National Institutes of Health Consensus Development Conference. Arch Neurol 1988; 45: 575-578. 
4 Davies PBD. Diffuse pulmonary involvement in von Recklinhausen's disease: a new syndrome. Thorax 1963; 18: 198.

5 Burkhalter JL, Morano JU, McCay MB. Diffuse interstitial lung disease in neurofibromatosis. South Med J 1986; 79: 944-946.

6 Davis SA, Kaplan RL. Neurofibromatosis and interstitial lung disease. Arch Dermatol 1978; 114: 1368-1369.

7 Davison LM. Neurofibromatosis with diffuse interstitial pulmonary fibrosis and phaeochromocytoma. $\mathrm{Br} J$ Radiol 1967; 40: 549-551.

8 De Scheerder I, Elinck W, Van Renterghem D, Cuvelier C, Tasson J, Van der Straeten M. Desquamative interstitial pneumonia and scar cancer of the lung complicating generalised neurofibromatosis. Eur J Respir Dis 1984; 65: 623-626.

9 Hardcastle SW, Hendricks ML. Neurofibromatosis (von Recklinghausen's disease): an unusual cause of parenchymal lung disease. A case report. S Afr Med J 1984; 66: 959-960.

10 Massaro D, Katz S. Fibrosing alveolitis: its occurrence, roentgenographic, and pathologic features in von Recklinghausen's neurofibromatosis. Am Rev Respir Dis 1966; 93: 934-942.

11 Massaro D, Katz S, Matthews MJ, Higgins G. Von Recklinghausen's neurofibromatosis associated with cystic lung disease. Am J Med 1965; 38: 233-240.

12 McCaffrey JT. Interstitial pulmonary fibrosis in von Recklinghausen's neurofibromatosis: report of case. J Am Osteopath Assoc 1982; 81: 688-691.

13 Meyer FJ, Teschler H, Schnabel R, Costabel U. Bronchoalveolar lavage cytology in pulmonary fibrosis associated with neurofibromatosis. Respir Med 1996; 90: 365-367.
14 Patchefsky AS, Atkinson WG, Hoch WS, Gordon G, Lipshitz HI. Interstitial pulmonary fibrosis and von Recklinghausen's disease. An ultrastructural and immunofluorescent study. Chest 1973; 64: 459-464.

15 Porterfield JK, Pyeritz RE, Traill TA. Pulmonary hypertension and interstitial fibrosis in von Recklinghausen neurofibromatosis. Am J Med Genet 1986; 25: 531-535.

16 Sagel SS, Forrest JV, Askin FB. Interstitial lung disease in neurofibromatosis. South Med J 1975; 68: 647-649.

17 Webb WR, Goodman PC. Fibrosing alveolitis in patients with neurofibromatosis. Radiology 1977; 122: 289-293.

18 Yokoyama A, Kohno N, Sakai K, Kondo K, Hirasawa Y, Hiwada K. Distal acinar emphysema and interstitial pneumonia in a patient with von Recklinghausen's disease: five-year observation following quitting smoking. Intern Med 1997; 36: 413-416.

19 American Thoracic Society, European Respiratory Society. American Thoracic Society/European Respiratory Society International Multidisciplinary Consensus Classification of the Idiopathic Interstitial Pneumonias. Am J Respir Crit Care Med 2002; 165: 277-304.

20 Ryu JH, Myers JL, Capizzi SA, Douglas WW, Vassallo R, Decker PA. Desquamative interstitial pneumonia and respiratory bronchiolitis-associated interstitial lung disease. Chest 2005; 127: 178-184.

21 Fabricant RN, Todaro GJ. Increased serum levels of nerve growth factor in von Recklinghausen's disease. Arch Neurol 1981; 38: 401-405.

22 Micera A, Vigneti E, Pickholtz D, et al. Nerve growth factor displays stimulatory effects on human skin and lung fibroblasts, demonstrating a direct role for this factor in tissue repair. Proc Natl Acad Sci USA 2001; 98: 6162-6167. 